\title{
Non-targeted LC-MS metabolomics approach for metabolic profiling of plasma and urine from pigs fed branched chain amino acids for maximum growth performance
}

Elham A. Soumeh ${ }^{1 *}$, Mette S. Hedemann ${ }^{1}$, Hanne D. Poulsen ${ }^{1}$, Etienne Corrent ${ }^{2}$, Jacob van Milgen $^{3}$, and Jan V. Nørgaard ${ }^{1, *}$

${ }^{1}$ Dept. of Animal Science, Aarhus University, Foulum, DK-8830 Tjele, Denmark; ${ }^{2}$ Ajinomoto Eurolysine S.A.S., F-75817 Paris Cedex 17, France; ${ }^{3}$ INRA, UMR1348 PEGASE, F-35590 Rennes, France.

Contents

Table S1. Analyzed composition of experimental diets with different Ile content ( $\mathrm{g} / \mathrm{kg}$, as-fed)................ S2

Table S2. Analyzed composition of experimental diets with different Val content ( $\mathrm{g} / \mathrm{kg}$, as-fed). ............... S3

Table S3. Analyzed composition of experimental diets with different Leu content (g/kg, as-fed)............... S4

Table S4. Effect of standardized ileal digestible (SID) Ile:Lys supply on performance of the pigs. .............. S5

Table S5. Effect of the standardized ileal digestible (SID) Val:Lys supply on performance of pigs.............. S6

Table S6. Effect of the standardized ileal digestible (SID) Leu:Lys supply on performance of the pigs. ...... S7

Figure S1. PCA score plots of groupings of all blood samples, blanks and quality controls from Ile (a), Val (b), and Leu (c) studies

Figure S2. PCA score plots of groupings of all urine samples, blanks and quality controls from Ile (a), Val (b), and Leu (c) studies S10 
Table S1. Analyzed composition of experimental diets with different Ile content (g/kg, as-fed $)^{1}$.

\begin{tabular}{|c|c|c|c|c|c|c|}
\hline \multirow[b]{2}{*}{ Item } & \multicolumn{6}{|c|}{ SID $^{2}$ Ile:Lys } \\
\hline & 0.42 & 0.46 & 0.50 & 0.54 & 0.58 & 0.62 \\
\hline Crude protein $(\mathrm{N} \times 6.25)$ & 163.9 & 171.2 & 168.8 & 168.6 & 166.4 & 168.6 \\
\hline Alanine & 5.6 & 5.8 & 5.7 & 5.7 & 5.6 & 5.6 \\
\hline Arginine & 8.2 & 8.5 & 8.3 & 8.3 & 8.1 & 8.2 \\
\hline Aspartate & 11.0 & 11.1 & 11.3 & 11.3 & 10.9 & 11.0 \\
\hline Cystine & 2.7 & 2.7 & 2.7 & 2.7 & 2.7 & 2.7 \\
\hline Glutamate & 34.4 & 34.6 & 34.0 & 33.5 & 32.6 & 32.4 \\
\hline Glycine & 5.7 & 5.9 & 5.8 & 5.8 & 5.7 & 5.7 \\
\hline Histidine & 4.4 & 4.5 & 4.5 & 4.5 & 4.4 & 4.4 \\
\hline Isoleucine & 5.6 & 6.2 & 6.4 & 6.9 & 7.1 & 7.7 \\
\hline Leucine & 13.3 & 13.5 & 13.1 & 13.1 & 12.7 & 12.8 \\
\hline Lysine & 12.2 & 12.8 & 12.4 & 12.5 & 11.7 & 12.2 \\
\hline Methionine & 4.3 & 4.7 & 4.5 & 4.6 & 4.3 & 4.4 \\
\hline Methionine + Cystine & 7.0 & 7.4 & 7.2 & 7.2 & 7.0 & 7.0 \\
\hline Phenylalanine & 8.5 & 8.8 & 8.7 & 8.7 & 8.4 & 8.5 \\
\hline Proline & 10.5 & 10.6 & 10.6 & 10.6 & 10.6 & 10.6 \\
\hline Serine & 6.9 & 7.1 & 7.1 & 7.1 & 6.9 & 7.0 \\
\hline Threonine & 7.9 & 8.3 & 8.1 & 8.2 & 7.9 & 7.9 \\
\hline Valine & 9.8 & 10.1 & 9.7 & 9.8 & 9.4 & 9.5 \\
\hline
\end{tabular}

\footnotetext{
Three samples of each diet were analyzed.

${ }^{2} \mathrm{SID}=$ standardized ileal digestible.
} 
Table S2. Analyzed composition of experimental diets with different Val content (g/kg, as-fed) ${ }^{1}$.

\begin{tabular}{|c|c|c|c|c|c|c|}
\hline \multirow[b]{2}{*}{ Item } & \multicolumn{6}{|c|}{ SID Val:Lys $^{2}$} \\
\hline & 0.58 & 0.62 & 0.66 & 0.70 & 0.74 & 0.78 \\
\hline Crude protein $(\mathrm{N} \times 6.25)$ & 178 & 181 & 182 & 179 & 180 & 180 \\
\hline Lys & 11.8 & 12.2 & 12.2 & 12.0 & 12.0 & 12.1 \\
\hline Met & 4.2 & 4.3 & 4.3 & 4.3 & 4.2 & 4.3 \\
\hline Met + Cys & 7.3 & 7.4 & 7.3 & 7.3 & 7.2 & 7.3 \\
\hline Ile & 7.1 & 7.2 & 7.2 & 7.1 & 7.1 & 7.2 \\
\hline Leu & 12.9 & 13.1 & 13.0 & 12.9 & 12.9 & 13.0 \\
\hline Val & 7.4 & 7.9 & 8.3 & 8.7 & 9.1 & 9.6 \\
\hline Thr & 8.2 & 8.3 & 8.2 & 8.2 & 8.2 & 8.2 \\
\hline Phe & 8.4 & 8.5 & 8.5 & 8.4 & 8.4 & 8.5 \\
\hline His & 4.4 & 4.5 & 4.5 & 4.4 & 4.4 & 4.4 \\
\hline Ala & 6.7 & 6.7 & 6.7 & 6.7 & 6.6 & 6.7 \\
\hline Arg & 9.9 & 9.9 & 10.0 & 9.9 & 9.8 & 9.9 \\
\hline Asp & 13.7 & 13.9 & 13.9 & 13.7 & 13.7 & 13.8 \\
\hline Glu & 39.4 & 39.3 & 38.8 & 38.1 & 37.7 & 37.5 \\
\hline Gly & 6.7 & 6.8 & 6.8 & 6.7 & 6.7 & 6.8 \\
\hline Pro & 11.5 & 11.6 & 11.6 & 11.5 & 11.5 & 11.6 \\
\hline Ser & 8.2 & 8.3 & 8.3 & 8.2 & 8.2 & 8.3 \\
\hline
\end{tabular}

\footnotetext{
Three samples of each diet were analyzed.

${ }^{2} \mathrm{SID}=$ standardized ileal digestible.
} 
Table S3. Analyzed composition of experimental diets with different Leu content (g/kg, as-fed) ${ }^{1}$.

\begin{tabular}{|c|c|c|c|c|c|c|}
\hline \multirow{2}{*}{ Item } & \multicolumn{6}{|c|}{ SID $^{2}$ Leu:Lys } \\
\hline & 0.70 & 0.80 & 0.90 & 1.00 & 1.10 & 1.20 \\
\hline Crude protein $(\mathrm{N} \times 6.25)$ & 157.3 & 155.8 & 153.8 & 158.3 & 155.1 & 158.5 \\
\hline Lys & 11.77 & 11.77 & 10.72 & 11.89 & 11.36 & 11.60 \\
\hline Met & 4.51 & 4.48 & 4.29 & 4.57 & 4.46 & 4.52 \\
\hline Met + Cys & 7.20 & 7.17 & 6.98 & 7.28 & 7.14 & 7.22 \\
\hline Thr & 7.90 & 8.00 & 7.73 & 8.07 & 7.90 & 7.99 \\
\hline Ile & 7.01 & 7.06 & 6.80 & 7.07 & 6.95 & 7.11 \\
\hline Leu & 8.87 & 9.93 & 10.72 & 12.28 & 13.01 & 14.27 \\
\hline Val & 9.25 & 9.33 & 9.03 & 9.47 & 9.21 & 9.34 \\
\hline Phe & 9.28 & 9.35 & 9.09 & 9.37 & 9.28 & 9.37 \\
\hline His & 4.36 & 4.36 & 4.18 & 4.39 & 4.29 & 4.39 \\
\hline Ala & 5.03 & 5.06 & 4.98 & 5.08 & 5.01 & 5.11 \\
\hline Arg & 7.17 & 7.24 & 7.09 & 7.29 & 7.18 & 7.35 \\
\hline Asp & 9.39 & 9.44 & 9.18 & 9.58 & 9.45 & 9.63 \\
\hline Glu & 35.17 & 34.41 & 33.06 & 32.40 & 31.04 & 30.46 \\
\hline Gly & 5.22 & 5.24 & 5.17 & 5.28 & 5.20 & 5.29 \\
\hline Pro & 10.03 & 10.08 & 10.15 & 10.10 & 10.08 & 10.17 \\
\hline Ser & 5.90 & 5.94 & 5.86 & 6.00 & 594 & 6.02 \\
\hline
\end{tabular}

${ }^{\top}$ Three samples of each diet were analyzed.

${ }^{2} \mathrm{SID}=$ standardized ileal digestible. 
Table S4. Effect of standardized ileal digestible (SID) Ile:Lys supply on performance of the pigs ${ }^{1}$.

\begin{tabular}{|c|c|c|c|c|c|c|c|c|c|}
\hline \multirow{2}{*}{ Item } & \multicolumn{7}{|c|}{ SID Ile:Lys } & \multicolumn{2}{|c|}{$P$-value ${ }^{3}$} \\
\hline & 0.42 & 0.46 & 0.50 & 0.54 & 0.58 & 0.62 & $\mathrm{SEM}^{2}$ & Linear & Quadratic \\
\hline Initial $\mathrm{BW}, \mathrm{kg}$ & 8.72 & 8.54 & 8.56 & 8.61 & 8.58 & 8.46 & 0.38 & 0.25 & 0.89 \\
\hline Final BW, kg & 14.15 & 14.46 & 15.17 & 15.02 & 14.79 & 14.68 & 0.32 & 0.08 & 0.007 \\
\hline ADFI d 0-14, g & 552 & 595 & 656 & 651 & 618 & 597 & 30 & 0.11 & 0.001 \\
\hline ADG d 0-14, g & 398 & 420 & 470 & 459 & 443 & 435 & 22 & 0.08 & 0.007 \\
\hline FCR d 0-14 & 1.41 & 1.40 & 1.40 & 1.42 & 1.38 & 1.38 & 0.03 & 0.50 & 0.62 \\
\hline \multicolumn{10}{|c|}{${ }^{1}$ Data represents the least square means of 16 pigs per treatment. } \\
\hline \multicolumn{10}{|c|}{${ }^{2}$ Standard error of mean. } \\
\hline
\end{tabular}


Table S5. Effect of the standardized ileal digestible (SID) Val:Lys supply on performance of pigs ${ }^{1}$.

\begin{tabular}{|c|c|c|c|c|c|c|c|c|c|}
\hline \multirow{2}{*}{ Item } & \multicolumn{6}{|c|}{ SID Val:Lys } & \multirow{2}{*}{$\mathrm{SEM}^{2}$} & \multicolumn{2}{|c|}{$P$-value ${ }^{3}$} \\
\hline & 0.58 & 0.62 & 0.66 & 0.70 & 0.74 & 0.78 & & Linear & Quadratic \\
\hline Initial BW, kg & 8.40 & 8.41 & 8.50 & 8.41 & 8.36 & 8.42 & 0.32 & - & - \\
\hline Final BW, kg & 12.67 & 13.08 & 14.00 & 14.33 & 13.70 & 14.41 & 0.48 & 0.002 & 0.16 \\
\hline ADFI d 0-14, g & 444 & 483 & 539 & 566 & 513 & 573 & 22 & $<0.001$ & 0.09 \\
\hline ADG d 0-14, g & 304 & 334 & 399 & 423 & 378 & 428 & 25 & $<0.001$ & 0.06 \\
\hline G:F d 0-14, g/g & 0.68 & 0.72 & 0.74 & 0.75 & 0.75 & 0.75 & 0.02 & 0.002 & 0.07 \\
\hline \multicolumn{10}{|c|}{${ }^{1} 16$ pigs were used for each treatment. The least square means are presented. Superscripts indicate } \\
\hline \multicolumn{10}{|c|}{${ }^{2}$ Standard error of mean. } \\
\hline${ }^{3}$ Orthogonal pol & mial & ntrast & efficie & s were & ised to & termin & linear & ad quadr & tic effects o \\
\hline
\end{tabular}


Table S6. Effect of the standardized ileal digestible (SID) Leu:Lys supply on performance of the pigs ${ }^{1}$.

\begin{tabular}{|c|c|c|c|c|c|c|c|c|c|}
\hline \multirow{2}{*}{ Item } & \multicolumn{6}{|c|}{ SID Leu:Lys } & \multirow{2}{*}{$\mathrm{SEM}^{2}$} & \multicolumn{2}{|c|}{$P$-value ${ }^{3}$} \\
\hline & 0.70 & 0.80 & 0.90 & 1.00 & 1.10 & 1.20 & & Linear & Quadratic \\
\hline Initial BW, kg & 7.77 & 7.69 & 7.78 & 7.79 & 7.79 & 7.82 & 0.68 & - & - \\
\hline Final BW, kg & 10.76 & 11.74 & 12.11 & 12.17 & 12.18 & 12.27 & 0.32 & 0.001 & 0.02 \\
\hline ADFI d $0-14, \mathrm{~g} / \mathrm{d}$ & 359 & 399 & 456 & 456 & 454 & 479 & 25 & $<0.001$ & 0.13 \\
\hline ADG d 0-14, g/d & 212 & 281 & 308 & 312 & 313 & 320 & 23 & $<0.001$ & 0.02 \\
\hline $\mathrm{G}: \mathrm{F} \mathrm{d} 0-14, \mathrm{~g} / \mathrm{g}$ & 0.60 & 0.72 & 0.69 & 0.69 & 0.69 & 0.66 & 0.03 & 0.29 & $<0.001$ \\
\hline
\end{tabular}

\footnotetext{
${ }^{1} 16$ pigs were used for each treatment. The least squares means are presented. Within a row, means without a common superscript differ $(P<0.05)$.

${ }^{2}$ Standard error of mean.

${ }^{3}$ Orthogonal polynomial contrast coefficients were used to determine linear and quadratic effects of increasing ratios of standardized ileal digestible Leu:Lys.
} 
Online Supporting Information
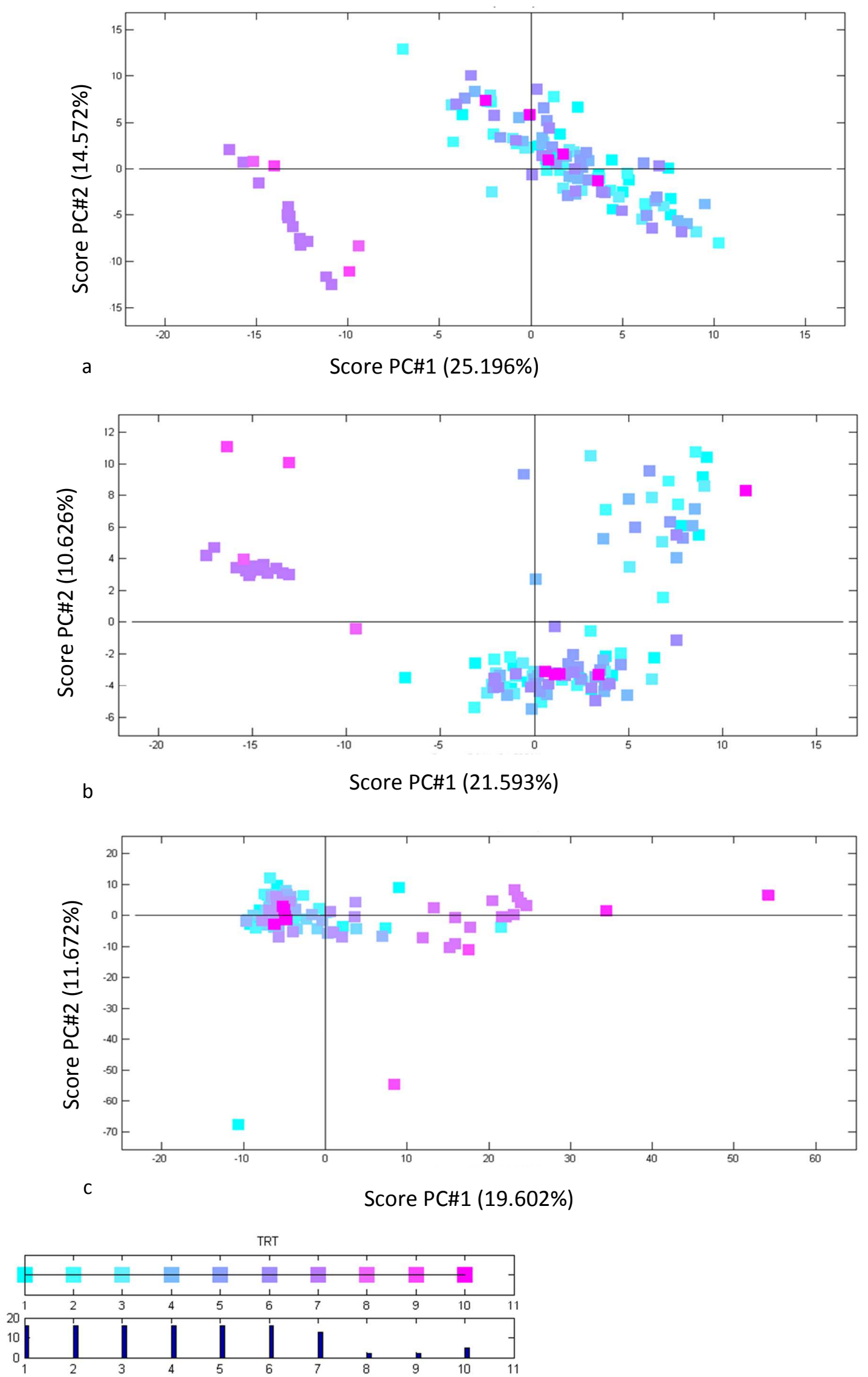


\section{Online Supporting Information}

Figure S1. PCA score plots of groupings of all blood samples, blanks and quality controls from Ile (a), Val (b), and Leu (c) studies. The 10 color codes for treatments (TRT) corresponds to 6 dietary level of each BCAA in the Ile, Val, and Leu studies; 7 is blank including internal standards, 8 and 9 are blanks, and 10 is quality control (a pooled sample of all blood samples). The 6 TRT were 0.42 , 0.46, 0.50, 0.54, 0.58, and 0.62 SID Ile:Lys, 0.58, 0.62, 0.66, 0.70, 0.74, and 0.78 SID Val:Lys, and 0.70, 0.80, 0.90, 1.00, 1.10, and 1.20 of SID Leu:Lys in Ile, Val, and Leu dose-response studies, respectively. 
Online Supporting Information

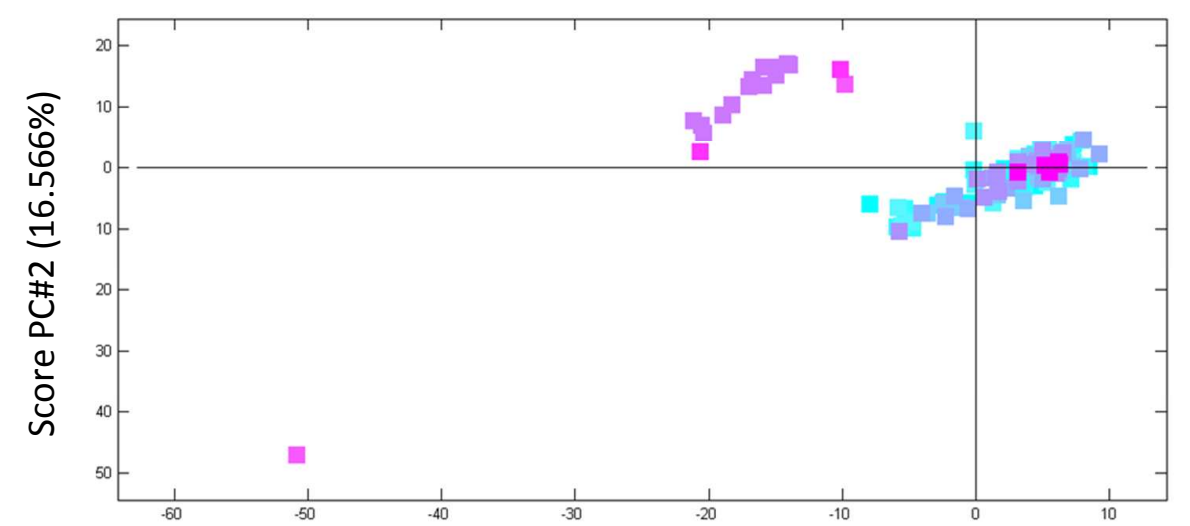

a

Score PC\#1 (25.046\%)
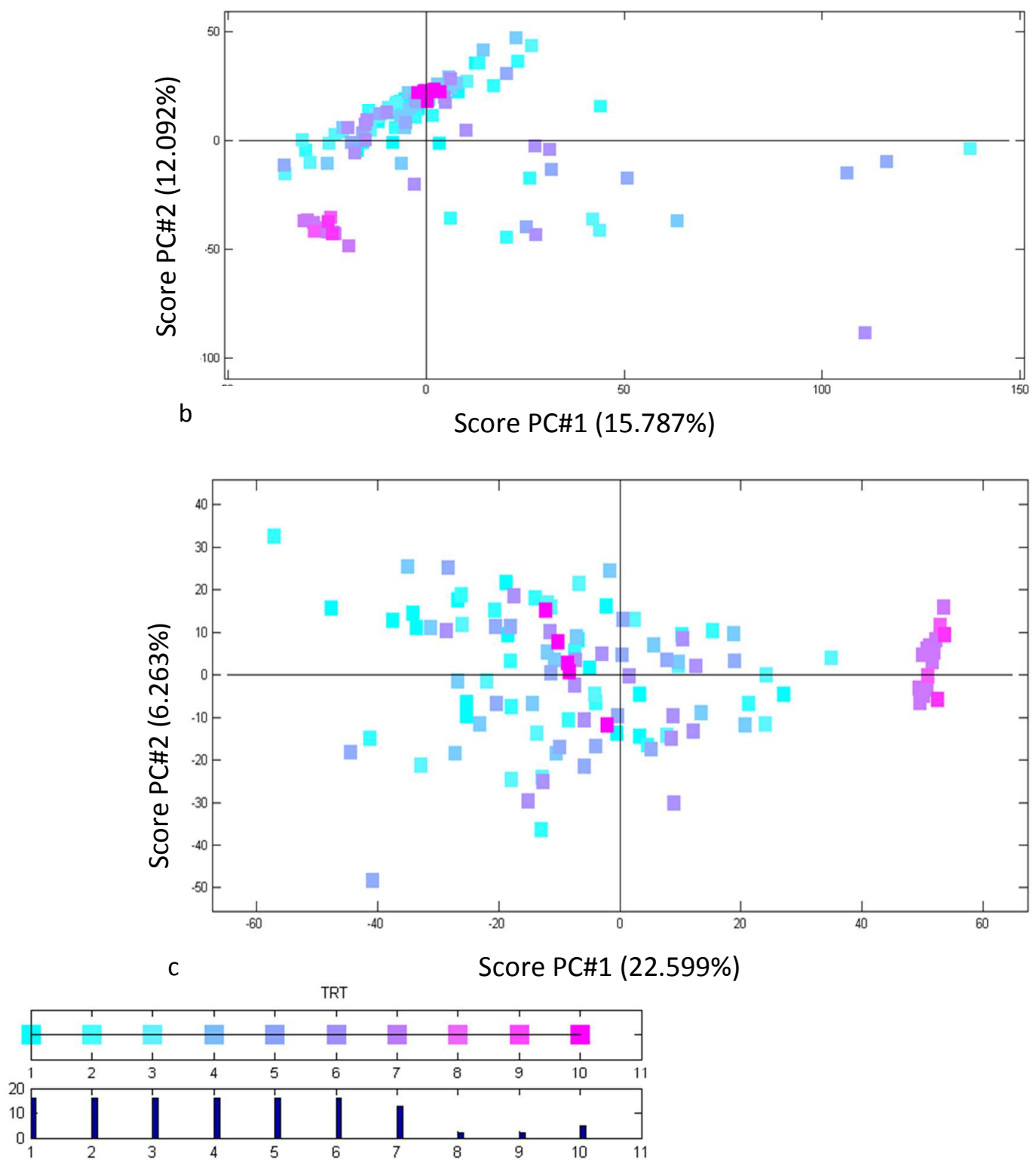


\section{Online Supporting Information}

Figure S2. PCA score plots of groupings of all urine samples, blanks and quality controls from Ile (a), Val (b), and Leu (c) studies. The 10 color codes for treatments (TRT) corresponds to 6 dietary level of each BCAA in the Ile, Val, and Leu studies; 7 is blank including internal standards, 8 and 9 are blanks, and 10 is quality control (a pooled sample of all urine samples). The 6 TRT were 0.42 , 0.46, 0.50, 0.54, 0.58, and 0.62 SID Ile:Lys, 0.58, 0.62, 0.66, 0.70, 0.74, and 0.78 SID Val:Lys, and $0.70,0.80,0.90,1.00,1.10$, and 1.20 of SID Leu:Lys in Ile, Val, and Leu dose-response studies, respectively. 